NASA Technical Memorandum 103858

\title{
A Theoretical Approach for Analyzing the Restabilization of Wakes
}

D. C. Hill, Ames Research Center, Moffett Field, California

April 1992

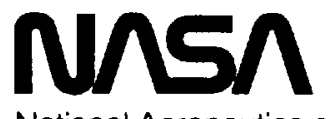

National Aeronautics and

Space Administration 
• 


\section{Summary}

Recently reported experimental results demonstrate that restabilization of the low-Reynolds-number flow past a circular cylinder can be achieved by the placement of a smaller cylinder in the wake of the first at particular locations. Traditional numerical procedures for modeling such phenomena are computationally expensive. An approach is presented here in which the properties of the adjoint solutions to the linearized equations of motion are exploited to map quickly the best positions for the small cylinder's placement. Comparisons with experiment and previous computations are favorable. The approach is shown to be applicable to general flows, illustrating how strongly control mechanisms that involve sources of momentum couple to unstable (or stable) modes of the system.

\section{Introduction}

We present here a linear analysis that permits, through use of stability theory, an approximation to the shift in the stability characteristics of steady, two-dimensional (2D) flow past a circular cylinder that occurs as a resuit of the presence of an additional small cylinder within the flow. The flow under study is incompressible and viscous, but the analysis could undoubtedly be generalized to include compressibility as well as other effects. For this analysis the feedback mechanism is provided by the small cylinder, which in effect adds momentum to the fluid. Other types of control laws based upon unsteady momentum addition could be modeled using the kind of analysis presented here and applied to three-dimensional (3D) flows.

The global dynamics of a system can be modified by specifying external sources which act in a way that depends upon the state of the system. The natural modes of the new system will differ from those of the old. If the system is not too highly distorted by the modification, we might expect to find that the natural frequencies of the new system have not been changed greatly. For linear waves in a continuous medium, adjoint solutions provide a means of examining in a simple fashion the effect of a particular feedback mechanism upon a particular mode of the system. Should the mode be unstable, then the adjoint solution has particular use in pinpointing a mechanism for its restabilization. The formulation of the adjoint linear problem is a classical method in differential equations, but because it is central to the calculation we take time here to outline its construction with special reference to the incompressible Navier-Stokes equations.
Viscous, incompressible, 2D flow past a circular cylinder is unstable above a critical Reynolds number of about 45 based upon cylinder diameter, and stable below the critical condition. The problem solved here is for a Reynolds number of 50 , and the regular and adjoint eigensolutions are found for the unstable mode using a modification of the spectral approach of Zebib (ref. 1). The unstable waves in this case are believed to grow up to form the time-periodic Karman vortex street. Strykowski and Sreenivasan (ref. 2) performed both experimental and numerical examinations of the effect of introducing a smaller cylinder at various points within the wake of a larger one past which the flow is unstable. Suitable positioning of the smaller cylinder was shown by both experimental observation and direct numerical simulation to suppress the onset of the vortex street. We present a simpler means of modeling the effect of the small cylinder in the wake of the first and demonstrate the restabilizing and, indeed, destabilizing effect which the small cylinder can have, by use of the adjoint to the unstable mode. The results of reference 2 are essentially verified, and furthermore there is a suggestion that there may be additional regions, overlooked in reference 2 , where the small cylinder could be positioned to restabilize the flow.

This research was supported by the National Research Council.

\section{Adjoint Eigensolutions}

Adjoint solutions are used in the study of partial differential equations (ref. 3), and find particular use in bifurcation theory (ref. 4). In this section the relationships between linearized solutions and their adjoints are outlined within the framework of the incompressible Navier-Stokes equations, and their basic mathematical properties are presented. The results in this section are not mathematically new, but are included for completeness.

Let us consider the steady, 2D, incompressible viscous flow field past a circular cylinder. (The application of this approach to time-periodic flows is covered in reference 5.) The velocity field, $\underline{V}(\underline{r})$, is normalized by the uniform flow at infinity, $V_{\infty}$, and pressure, $P(\underline{r})$, normalized by $\rho V_{\infty}{ }^{2}$, where $\rho$ is the fluid density. If $V_{i}$ is the component of $\underline{V}$ in the ith coordinate direction, then we have for the equations of motion

$$
v_{j} \frac{\partial v_{i}}{\partial x_{j}}-\frac{2}{R} \frac{\partial^{2} v_{i}}{\partial x_{j}^{2}}+\frac{\partial P}{\partial x_{i}}=0
$$

and 


$$
\frac{\partial V_{i}}{\partial x_{i}}=0
$$

where $\mathrm{R}$ is the Reynolds number based upon cylinder diameter. Lengths are scaled on the cylinder radius. Numerically the problem is solved for the stream function on an annular domain centered on the cylinder center, using a spectral method as described in reference 1 . The reader is referred to this paper for details of the computational method.

The flow shown in figure 1 is the stream function, $\Psi$, for a Reynolds number of 50 , and has been constructed using 44 Chebychev polynomials in the radial direction and 40 Fourier components in the angular direction. The outer computational perimeter is at 20 cylinder radii. The steady velocity field is then $\underline{V}=\nabla \times(\Psi \underline{z})$, where $\underline{z}$ is a unit vector normal to the plane of the $2 \mathrm{D}$ flow. The separation angle and the wake length agree with the results of reference 6 .

If we superimpose linear time-dependent disturbances upon this field, then they satisfy

$$
\frac{\partial \tilde{\mathrm{v}}_{\mathrm{i}}}{\partial \mathrm{t}}+(\mathrm{L}(\underline{\mathrm{V}} ; \mathbf{R}) \underline{\underline{\underline{y}}})_{\mathrm{i}}+\frac{\partial \tilde{\mathrm{p}}}{\partial \mathrm{x}_{\mathrm{i}}}=0
$$

and

$$
\frac{\partial \tilde{v}_{i}}{\partial x_{i}}=0
$$

where $\tilde{v}_{i}$ is the ith component of the velocity disturbance, $\tilde{\underline{v}}$, and $\tilde{\mathrm{p}}$ is the pressure disturbance. Here the time variable is scaled by $r / V_{\infty}$, where $r$ is the cylinder radius. The ith component of the linear operator $L(\underline{V} ; R)$ is

$$
(L(\underline{V} ; R) \underline{\tilde{y}})_{i}=V_{j} \frac{\partial \tilde{v}_{i}}{\partial x_{j}}+\tilde{v}_{j} \frac{\partial V_{i}}{\partial x_{j}}-\frac{2}{R} \frac{\partial^{2} \tilde{v}_{i}}{\partial x_{j}^{2}}
$$

Taking the Fourier transforms in time,

$$
\underline{v}(\underline{r}, \omega)=\int_{-\infty}^{\infty} \underline{\underline{v}}(\underline{r}, t) e^{-i \omega t} d t, p(\underline{r}, \omega)=\int_{-\infty}^{\infty} \tilde{p}(\underline{r}, t) e^{-i \omega t} d t
$$

gives

$$
i \omega v_{i}+(L(\underline{V} ; R) \underline{v})_{i}+\frac{\partial p}{\partial x_{i}}=0
$$

and

$$
\frac{\partial v_{i}}{\partial x_{i}}=0
$$

Applying boundary conditions $v_{i}=0$ on the boundary of the computational domain defines an eigenvalue problem. (In our problem the conditions are applied on the cylinder surface and at 20 cylinder radii.)

We will now formulate the adjoint equations. Central to the creation of an adjoint solution is the definition of an inner product. For a pair of complex vector fields, $\underline{u}$ and $\underline{v}$, defined over the flow domain, we define the inner product to be

$$
[\underline{u}, \underline{v}]=\int_{v} \underline{u} \cdot \underline{v} d V
$$

after reference 7 . We now look for an operator $L^{*}(\underline{V} ; \mathbf{R})$ for which

$$
[\mathrm{L}(\underline{\mathrm{V}} ; \mathrm{R}) \underline{\mathrm{u}}, \underline{\mathrm{v}}]=\left[\underline{\mathrm{u}}, \mathrm{L}^{*}(\underline{\mathrm{V}} ; \mathrm{R}) \underline{\mathrm{u}}\right]
$$

for all $\underline{u}$ and $\underline{v}$, where $\underline{u}$ satisfies the boundary conditions for the regular linear problem and $\underline{x}$ has boundary conditions chosen to ensure the validity of equation (10).

From equations (7) and (8), the linear adjoint problem with respect to the inner product (eq. (9)) can now be formulated as

$$
i \omega^{*} v_{i}^{*}+\left(L^{*}(\underline{V} ; R) \underline{v}^{*}\right)_{i}-\frac{\partial p^{*}}{\partial x_{i}}=0
$$

and

$$
\frac{\partial v_{i}^{*}}{\partial x_{i}}=0
$$

With the boundary conditions chosen to ensure equation (10), the adjoint solutions can be solved to give a set of eigenfrequencies and eigensolutions. If $\mathrm{y}^{(\mathrm{n})}$ is the $\mathrm{nth}$ solution to equations (7) and (8) with eigenfrequency $\omega_{n}$, and $\underline{v}^{(m)^{*}}$ is the mth adjoint eigensolution to equations (11) and (12) with frequency $\omega_{\mathrm{m}}^{*}$, then it is easily shown that

$$
\left(\omega_{\mathrm{n}}-\omega_{\mathrm{m}}^{*}\right)\left[\underline{\mathrm{v}}^{(\mathrm{n})}, \underline{\mathrm{v}}^{(\mathrm{m})^{*}}\right]=0
$$

Consequently either the frequencies of the nth and $m$ th mode are the same, or the regular and adjoint solutions are orthogonal with respect to the inner product. We can relabel the frequencies, order them in increasing order of stability $\left(-\operatorname{Im}\left(\omega_{0}\right) \geq-\operatorname{Im}\left(\omega_{1}\right) \geq \ldots\right)$, and normalize the velocity fields so that

$$
\begin{aligned}
\omega_{n}=\omega_{n}^{*}, \text { and }\left[\underline{v}^{(n)}, \underline{v}^{(m)^{*}}\right] & =\delta_{n, m} \\
\text { for } n, m & =0,1,2, \ldots
\end{aligned}
$$


The adjoint linear operator $L^{*}(\underline{V} ; R)$ to the linearized Navier-Stokes equations operator $\mathbf{L}(\underline{\mathrm{V}} ; \mathrm{R})$ can be developed from

$$
\begin{aligned}
(\underline{L}(\underline{\mathrm{V}} ; \mathrm{R}) \underline{u})_{i} v_{i} & =\left(\mathrm{V}_{j} \frac{\partial u_{i}}{\partial x_{j}}+u_{j} \frac{\partial V_{i}}{\partial x_{j}}-\frac{2}{R} \frac{\partial^{2} u_{i}}{\partial x_{j}^{2}}\right) v_{i} \\
& =u_{i}\left(L^{*}(\underline{V} ; R) \underline{v}\right)_{i}+\frac{\partial f_{j}}{\partial x_{j}}
\end{aligned}
$$

where

$$
\left(L^{*}(\underline{V} ; R) \underline{v}\right)_{i}=-v_{j} \frac{\partial v_{i}}{\partial x_{j}}+v_{j} \frac{\partial v_{j}}{\partial x_{i}}-\frac{2}{R} \frac{\partial^{2} v_{i}}{\partial x_{j}^{2}}
$$

and

$$
f_{j}=v_{j} u_{i} v_{i}-\frac{2}{R}\left(v_{i} \frac{\partial u_{i}}{\partial x_{j}}-u_{i} \frac{\partial v_{i}}{\partial x_{j}}\right)
$$

Then

$$
[\mathrm{L}(\underline{\mathrm{V}} ; \mathrm{R}) \underline{\mathrm{u}}, \underline{\mathrm{v}}]=\left[\underline{\mathrm{u}}, \mathrm{L}^{*}(\underline{\mathrm{V}} ; \mathrm{R}) \underline{\mathrm{v}}\right]+\int_{\Gamma} \mathrm{f}_{\mathrm{j}} \mathrm{dn} j
$$

A no-slip condition on the cylinder surface and at the outer computational boundary for both the regular and adjoint velocity fields ensures that $f_{j}=0$.

At a Reynolds number of 50, the incompressible flow past a circular cylinder is just unstable, with the disturbance being asymmetric and the eigenfrequency being $\omega_{0}=0.376-i 0.000353$. The Strouhal number, based on cylinder diameter and with frequency $f$ in Hertz, is then $2 \mathrm{fr} / \mathrm{V}_{\infty}=\operatorname{Re}\left(\omega_{0} / \pi\right)=0.120$, as expected. The real and imaginary parts of the stream function are shown in figures 2(a) and 2(b). Here 34 Chebychev polynomials have been used in the radial direction, and 35 Fourier components in the angular direction. (These have been calculated using a modification of the method of reference 1 which is not described here.) This clearly resembles the forms found in reference 8 . Figures $3(a)$ and $3(b)$ show the corresponding adjoint solutions using the same number of coefficients. Most notable here is the occurrence of features upstream of the cylinder (the steady flow is from left to right), something not seen in the regular solutions. The adjoint solution is largest in the immediate wake of the cylinder.

\section{The Control of Unstable Flows}

The recent paper by Strykowski and Sreenivasan (ref. 2) documents the effect on overall system stability of placing a small cylinder in the wake of a larger cylinder past which the flow is unstable. We model the effect of the small cylinder as a pointwise supply of momentum to the flow equal and opposite to the anticipated drag. A quasi-steady approximation to the drag upon a small cylinder in a slowly varying flow is used, and is believed to be valid since the time scale of adjustment of the flow behind the small cylinder is shorter than that behind the large cylinder by at least a factor of the diameter ratio.

If $\underline{V}+\underline{\underline{v}}$ is the flow velocity in the vicinity of the small cylinder, then the drag vector is taken to be

$$
\underline{\tilde{d}}=\varepsilon(\underline{V}+\underline{\tilde{y}})|\underline{\mathrm{V}}+\underline{\tilde{y}}|
$$

where $\varepsilon$ represents $\rho C_{D} d / 2$ for the small cylinder, where $d$ is its diameter and $C_{D}$ is the effective drag coefficient. We assume $\varepsilon$ is small. Taking the Fourier transform in time of the drag, and expanding in powers of the velocity disturbance, we find that

$$
\underline{d}=\varepsilon\left(|\underline{v}| \underline{v} \delta(\omega)+\frac{\underline{v}}{|\underline{v}|} \underline{v} \cdot \underline{v}+|\underline{v}| \underline{v}\right)+0\left(|\underline{v}|^{2}\right)
$$

We model the presence of the small cylinder in the wake of the larger cylinder at position $\underline{r}_{0}$ as a momentum supply, which has both a steady and unsteady component, on the right-hand side of the momentum equations of strength

$$
-\varepsilon\left(|\underline{V}| \underline{V} \delta(\omega)+\frac{\underline{V}}{|\underline{V}|} \underline{V} \cdot \underline{v}+|\underline{V}| \underline{v}\right) \delta\left(\underline{r}-\underline{r}_{0}\right)
$$

If we write $\underline{\mathrm{V}}^{(0)}$ for the steady flow past the cylinder, satisfying equations (1) and (2), then the presence of the small cylinder will lead to a modification of the steady flow component. If we write the new steady flow as

$$
\underline{v}=\underline{v}^{(0)}+\varepsilon \underline{V}^{(1)}+0\left(\varepsilon^{2}\right)
$$

then it is not hard to show that we must have

$$
\mathrm{L}\left(\underline{\mathrm{V}}^{(0)} ; \mathrm{R}\right) \underline{\mathrm{V}}^{(1)}=-\left|\underline{\mathrm{V}}^{(0)}\right| \underline{\mathrm{V}}^{(0)} \delta\left(\underline{\mathrm{r}}-\underline{\mathrm{r}}_{0}\right)
$$

The linearized equations of unsteady motion around this new base flow are 


$$
\begin{aligned}
i \omega v_{i}+ & \left(L\left(\underline{\underline{v}}^{(0)} ; R\right) \underline{v}\right)_{i}+\frac{\partial p}{\partial x_{i}} \\
= & -\varepsilon\left(\frac{\underline{v}^{(0)}}{\left|\underline{v}^{(0)}\right|} \underline{v}^{(0)} \cdot \underline{v}+\left|\underline{v}^{(0)}\right| \underline{v}\right)_{i} \\
& \times \delta\left(\underline{\left.\underline{r}-\underline{r}_{0}\right)}-\varepsilon\left(v_{j}^{(1)} \frac{\partial v_{i}}{\partial x_{j}}+v_{j} \frac{\partial v_{i}^{(1)}}{\partial x_{j}}\right)+0\left(\varepsilon^{2}\right)\right.
\end{aligned}
$$

The choice of equation (21) for the momentum supply is specific to our present problem. Clearly this could represent other kinds of control mechanisms in which momentum is supplied to the system.

It is anticipated that there will be an eigenvalue of the inhomogeneous momentum equation (eq. (24)) that is close to the unstable eigenvalue for the flow past the large cylinder on its own. Consequently we write

$$
\underline{v}=\underline{v}^{(0)}+\varepsilon \sum_{n=1}^{\infty} b_{n} \underline{v}^{(n)}+0\left(\varepsilon^{2}\right)
$$

and

$$
\omega=\omega_{0}+\varepsilon \omega^{\prime}+0\left(\varepsilon^{2}\right)
$$

where $\underline{v}^{(0)}$ is the unstable eigensolution with eigenfrequency $\omega_{0}$, for some constants $b_{n}$ and $\omega$ '. Substituting into the inhomogeneous momentum equation and collecting terms of equal order in $\varepsilon$, to zeroth order, we retrieve the unmodified eigensolution as expected. To order $\varepsilon$,

$$
\begin{aligned}
i \omega^{\prime} \underline{\underline{v}}^{(0)} & +i \omega_{0} \sum_{n=1}^{\infty} b_{n} \underline{\underline{v}}^{(n)}+\sum_{n=1}^{\infty} b_{n} L \underline{\underline{v}}^{(n)} \\
= & -\left(\frac{\underline{\mathrm{v}}^{(0)}}{\left|\underline{\mathrm{V}}^{(0)}\right|} \underline{\mathrm{V}}^{(0)} \cdot \underline{\mathrm{v}}^{(0)}+\left|\underline{\mathrm{v}}^{(0)}\right| \underline{\underline{v}}^{(0)}\right) \delta\left(\underline{\mathrm{r}}-\underline{\mathrm{r}}_{0}\right) \\
& -\underline{\mathrm{v}}^{(1)} \cdot \nabla \underline{\underline{y}}^{(0)}-\underline{\mathrm{v}}^{(0)} \cdot \nabla \underline{\underline{v}}^{(1)}
\end{aligned}
$$

Multiplying the equation by the adjoint solution to $\underline{v}^{(0)}$ and integrating over space eliminates the summation factors because of the properties of the adjoints, revealing that

$i \omega^{\prime}=-\left(\frac{\underline{\mathrm{V}}^{(0)}}{\left|\underline{\mathrm{V}}^{(0)}\right|} \underline{\mathrm{V}}^{(0)} \cdot \underline{\mathrm{v}}^{(0)}+\left|\underline{\mathrm{V}}^{(0)}\right| \underline{\mathrm{v}}^{(0)}\right) \cdot \underline{\mathrm{v}}^{(0)^{*}}+\Delta$

where the term on the right-hand side in brackets is evaluated at the small-cylinder location $\underline{r}_{0}$, and

$$
\Delta=\int_{V} v_{j}^{(1)} v_{i}^{(0)} \frac{\partial v_{i}^{(0)^{*}}}{\partial x_{j}} d V
$$

The value of $\varepsilon \omega^{\prime}$ is the first-order correction to the eigenfrequency $\omega_{0}$ resulting from the placement of a small cylinder at position $r=\mathfrak{r} 0$. The shift in the damping rate is given by $-\varepsilon \operatorname{Im}\left(\omega^{\prime}\right)$; a negative shift encourages restabilization, whereas a positive shift enhances the instability.

From this point onward we ignore the influence upon the eigenvalue of the correction, $\underline{V}^{(1)}$, to the steady flow and set $\Delta$ equal to zero. Although this factor may not be insignificant, it is ignored here for the sake of simplicity. The result appears to justify this simplification, but the point requires further investigation.

The terms $-\operatorname{Im}\left(\omega^{\prime}\right)$ and $\operatorname{Re}\left(\omega^{\prime}\right)$ are plotted in figures 4 and 5 , respectively, (with $\Delta=0$ ). The demarcation of the stability boundary can be achieved only with some determination of $\varepsilon$. We do not attempt this, and restrict our interest purely to the relative merits of one position for the small cylinder versus another. Regions of large negative shift imply that placing the small cylinder in this area will have a strong stabilizing effect upon the flow. It is interesting to make the comparison with the hard-earned numerical results of reference 2 ; note that essentially the same features have appeared in our plot. Furthermore, an additional region where vortex shedding may be suppressed is indicated 7 to 9 cylinder diameters downstream. This was not reported in reference 2 . It is not entirely clear whether this is a physical effect or an artifact of the finite computational domain. The experimental results of reference 2 are for Reynolds numbers of order 80 and above, but show essentially the same features as the more negative contours of figure 4 .

A further feature that can be seen in figure 4 is the region adjacent to the main cylinder walls in which the instability is enhanced by the small cylinder. This is to be expected, because when the small cylinder is close to the large cylinder the effective Reynolds number is increased, thus encouraging the instability.

The map of the shift in the Strouhal number caused by the small cylinder shows regions where the frequency is increased, whereas in other regions it is decreased. It was observed in reference 2 that the vortex shedding eventually returns at higher Reynolds numbers, with the Strouhal number significantly reduced. In the regions of strong suppression indicated in figure 4 (contour levels of about -0.02 ), the corresponding frequency shift in figure 5 is negative. Therefore the results here are consistent with this frequency-reduction trend. 


\section{Conclusions}

It has been shown that an approximate linear stability analysis can be employed to illustrate how the stability of low-Reynolds-number flow past a circular cylinder is modified by the placement of a smaller cylinder in its wake. The results are shown to compare favorably with the more direct numerical computation of reference 2 . While the choice of "control mechanism" was mechanical in this configuration, linear stability analysis could be applied to more general types of control in which a fluid system receives an external supply of momentum according to some prescribed control law.

\section{References}

1. Zebib, A.: Stability of Viscous Flow past a Circular Cylinder. J. Eng. Math., vol. 21, 1987, pp. 155-165.

2. Strykowski, P. J.; and Sreenivasan, K. R.: On the Formation and Suppression of Vortex 'Shedding' at Low Reynolds Numbers. J. Fluid Mech., vol. 218, 1990, pp. 71-107.
3. Webster, A. G.: Partial Differential Equations of Mathematical Physics. Hafner Publishing Co., 1950.

4. Iooss, G.; and Joseph, D. D.: Elementary Stability and Bifurcation Theory. Springer-Verlag, 1980.

5. Joseph, D. D.: "Remarks about Bifurcation and Stability of Quasi-periodic Solutions which Bifurcate from Periodic Solutions of the Navier-Stokes Equations." Lecture Notes in Mathematics 322. Nonlinear Problems in the Physical Sciences and Biology, p. 130, Springer, 1972.

6. Dennis, S. C. R.; and Chang, G. Z.: Numerical Solutions for Steady Flow past a Circular Cylinder at Reynolds Numbers up to 100 . J. Fluid Mech., vol. 42, 1970, pp. 471-489.

7. Ladyzhenskaya, O. A.: The Mathematical Theory of Viscous Incompressible Flow. Second ed., Gordon and Breach, 1969.

8. Jackson, C. P.: A Finite-Element Study of the Onset of Vortex Shedding in Flow past Variously Shaped Bodies. J. Fluid Mech., vol. 182, 1987, pp. 23-45.

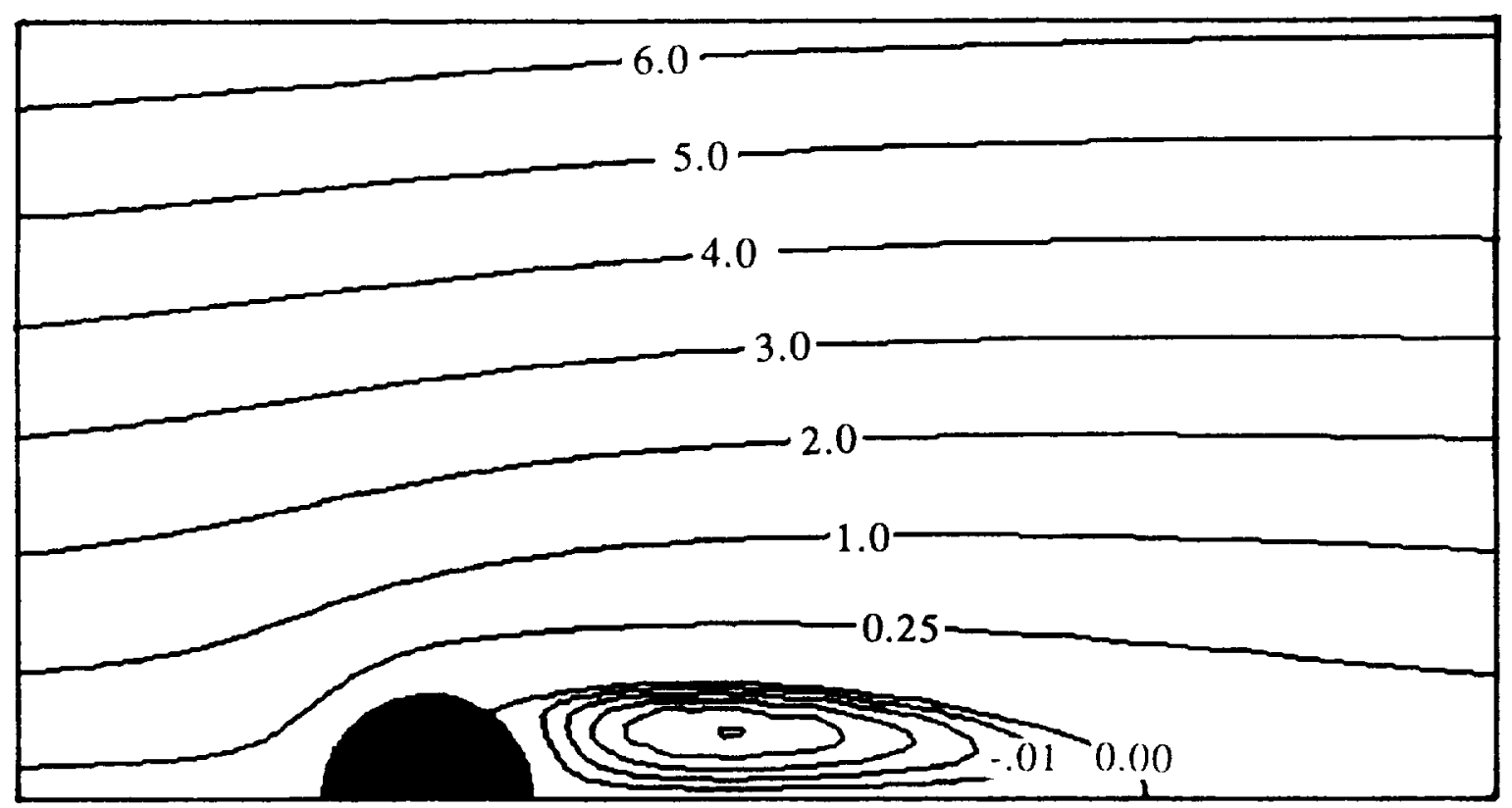

Figure 1. The steady stream function for viscous, incompressible flow past a circular cylinder at a Reynolds number of 50 . 


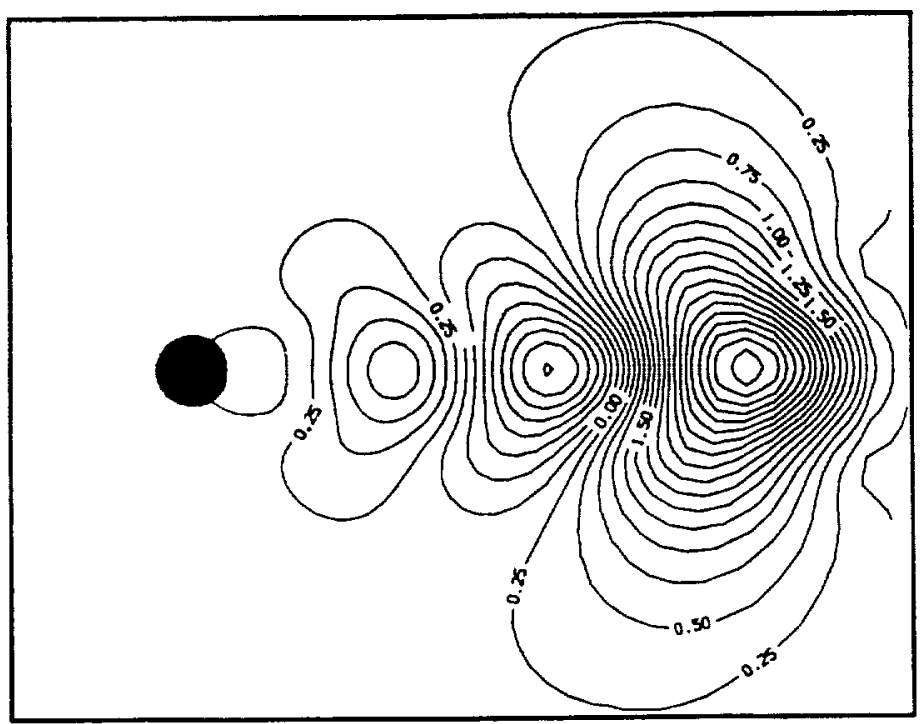

(a)

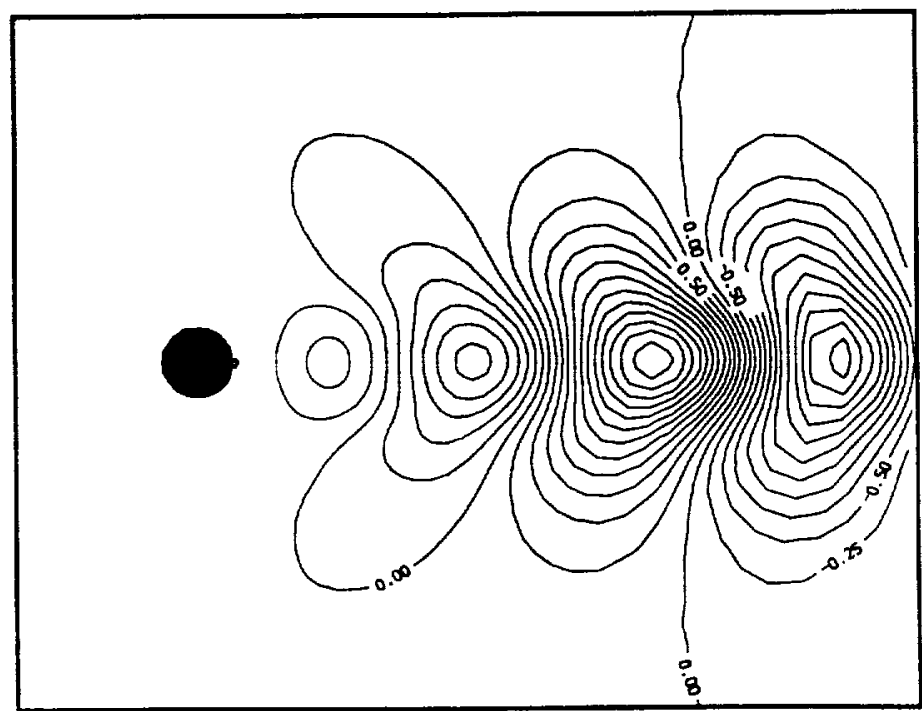

(b)

Figure 2. The (a) real and (b) imaginary parts of the stream function representing the unstable eigensolution for viscous, incompressible flow past a circular cylinder at a Reynolds number of 50. 


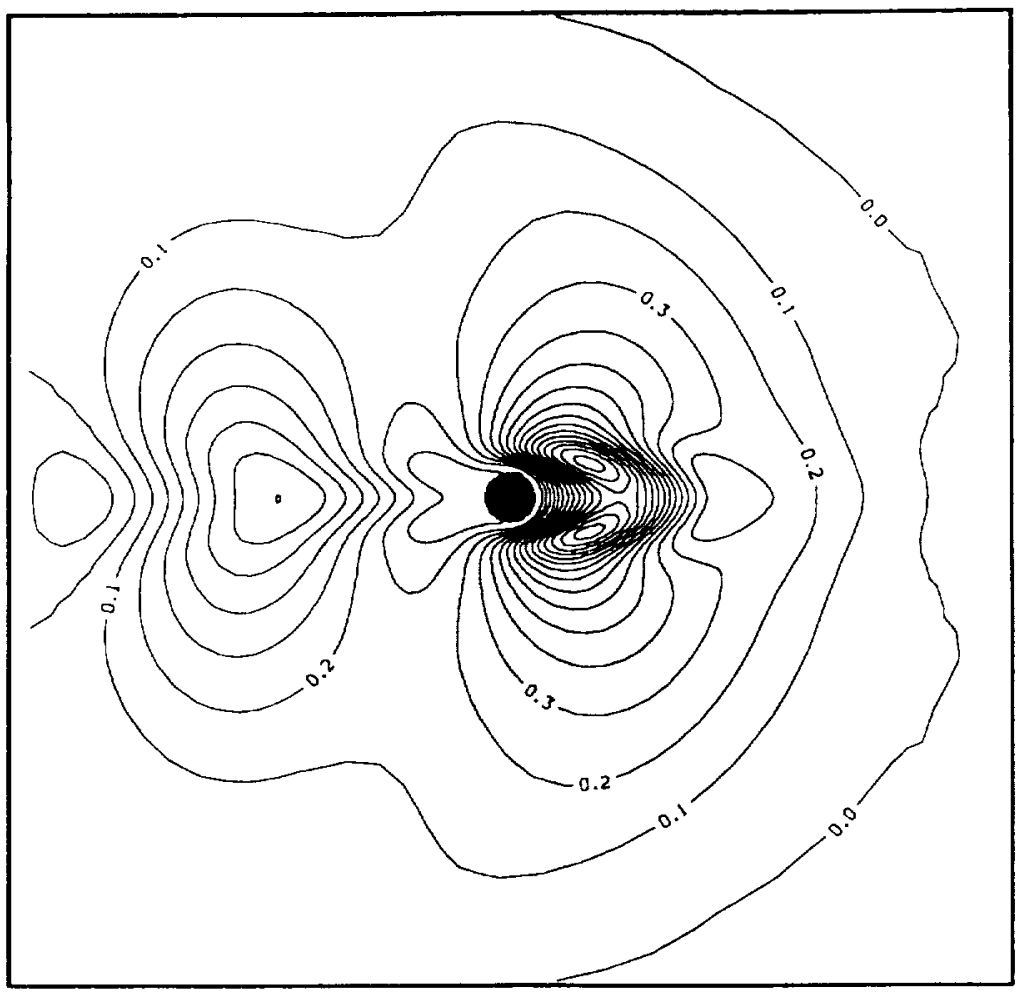

(a)

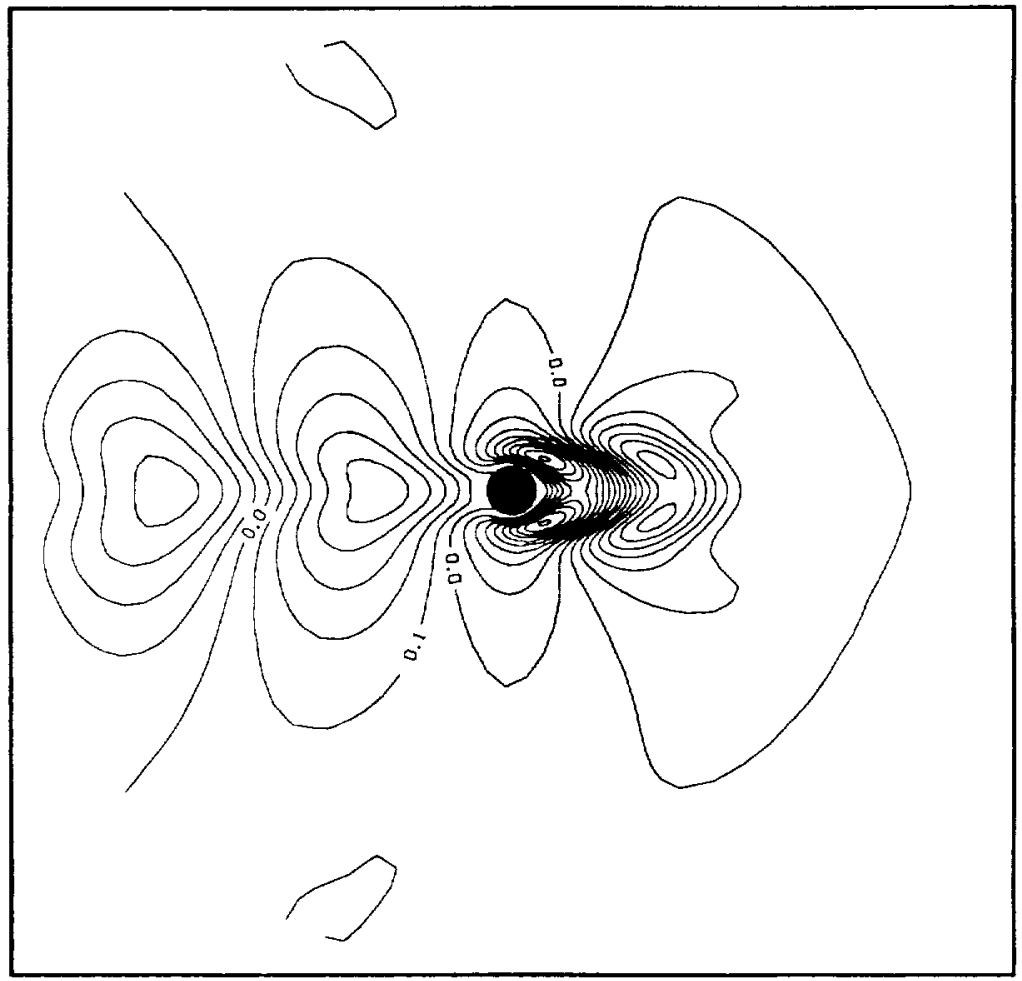

(b)

Figure 3. The (a) real and (b) imaginary parts of the adjoint stream function representing the unstable mode for flow past a circular cylinder at a Reynolds number of 50. 


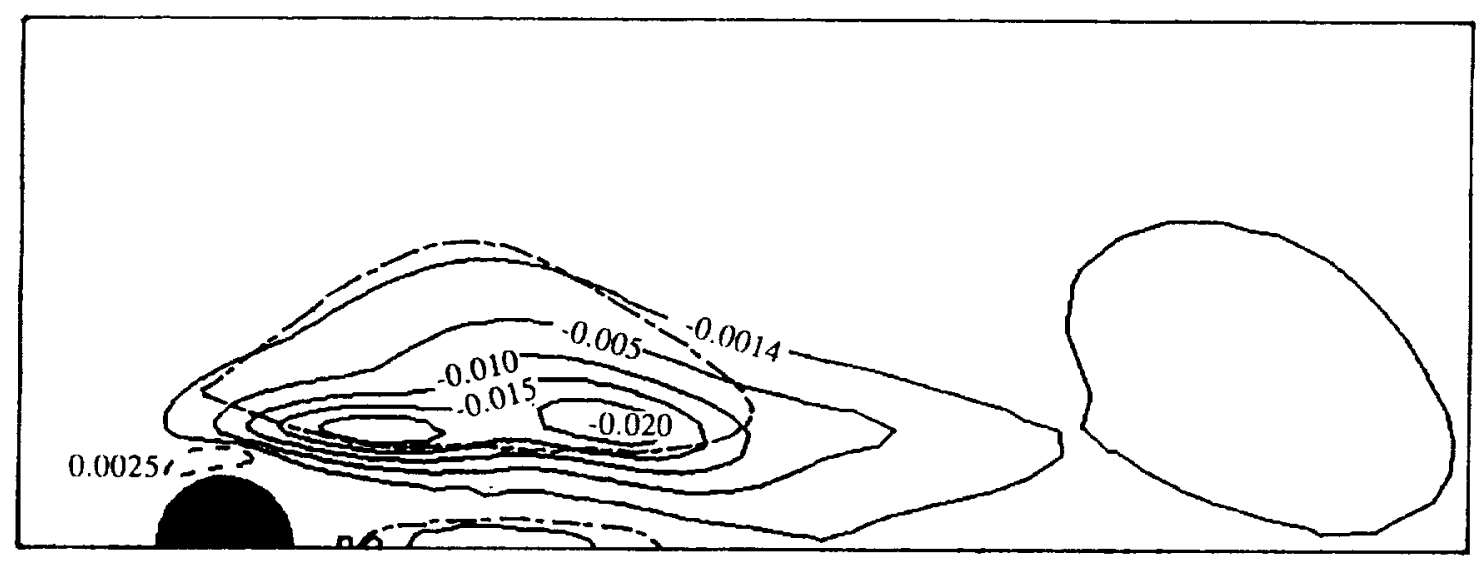

Figure 4. The shift in the damping rate, $-I m\left(\omega^{\prime}\right)$, as a function of the placement position for the small cylinder at a Reynolds number of 50 . The chain dash line shows the computed results of reference 2 for cylinder placement for neutral stability at a Reynolds number of 48 (diameter of small cylinder is $1 / 10$ that of large).

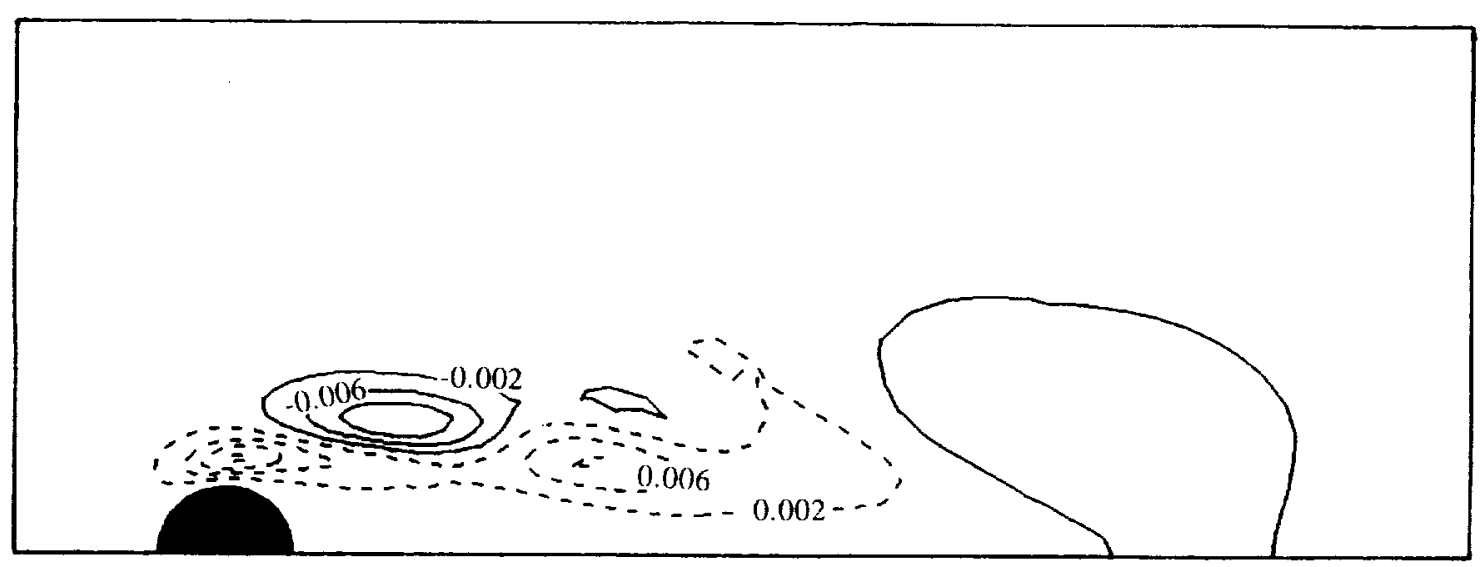

Figure 5. The shift in $R e\left(\omega^{\prime}\right)$ (proportional to the Strouhal number) as a function of the placement position for the small cylinder at a Reynolds number of 50. 


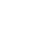




\begin{tabular}{|c|c|c|c|c|}
\hline \multicolumn{3}{|c|}{ REPORT DOCUMENTATION PAGE } & & $\begin{array}{l}\text { Form Approved } \\
\text { OMB No. 0704-0188 }\end{array}$ \\
\hline \multicolumn{5}{|c|}{ 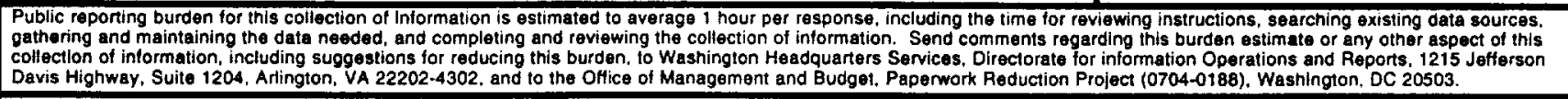 } \\
\hline 1. AGENCY USE ONLY (Leave blank) & k) $\begin{array}{l}\text { 2. REPORT DATE } \\
\text { April } 1992\end{array}$ & \multicolumn{3}{|c|}{$\begin{array}{l}\text { 3. AEPORT TYPE AND DATES COVERED } \\
\text { Technical Memorandum }\end{array}$} \\
\hline \multicolumn{3}{|c|}{$\begin{array}{l}\text { 4. TITLE AND SUBTITLE } \\
\text { A Theoretical Approach for Analyzing the Restabilization of Wakes }\end{array}$} & \multirow{2}{*}{\multicolumn{2}{|c|}{$\begin{array}{l}\text { 5. FUNDING NUMBERS } \\
505-59-53\end{array}$}} \\
\hline \multicolumn{3}{|l|}{$\begin{array}{l}\text { 6. AUTHOR(S) } \\
\text { D. C. Hill }\end{array}$} & & \\
\hline \multicolumn{3}{|c|}{$\begin{array}{l}\text { 7. PERFORMING ORGANIZATION NAME(S) AND ADDRESS(ES) } \\
\text { Ames Research Center } \\
\text { Moffett Field, CA } 94035-1000\end{array}$} & \multicolumn{2}{|c|}{$\begin{array}{l}\text { 8. PERFORMING OAGANIZATION } \\
\text { REPORT NUMBER }\end{array}$} \\
\hline \multicolumn{3}{|c|}{$\begin{array}{l}\text { National Aeronautics and Space Administration } \\
\text { Washington, DC 20546-0001 }\end{array}$} & \multicolumn{2}{|c|}{ NASA TM-103858 } \\
\hline \multicolumn{5}{|c|}{$\begin{array}{l}\text { 11. SUPPLEMENTAAY NOTES } \\
\text { Point of Contact: D. C. Hill, Ames Research Center, MS 227-4, Moffett Field, CA 94035-1000; } \\
\text { (415) } 6045855 \text { or FTS 464-5855 }\end{array}$} \\
\hline \multicolumn{3}{|c|}{$\begin{array}{l}\text { 12a. DISTRIBUTION/AVAILABILITY STATEMENT } \\
\text { Unclassified - Unlimited } \\
\text { Subject Category } 34\end{array}$} & \multicolumn{2}{|c|}{ 12b. DISTRIBUTION CODE } \\
\hline \multicolumn{5}{|c|}{$\begin{array}{l}\text { Recently reported experimental results demonstrate that restabilization of the low-Reynolds-number flow } \\
\text { past a circularcylinder can be achieved by the placement of a smaller cylinder in the wake of the first at particular } \\
\text { locations. Traditional numerical procedures for modeling such phenomena are computationally expensive. An } \\
\text { approach is presented here in which the properties of the adjoint solutions to the linearized equations of motion } \\
\text { are exploited to map quickly the best positions for the small cylinder's placement. Comparisons with } \\
\text { experiment and previous computations are favorable. The approach is shown to be applicable to general flows, } \\
\text { illustrating how strongly control mechanisms that involve sources of momentum couple to unstable (or stable) } \\
\text { modes of the system. }\end{array}$} \\
\hline \multirow{2}{*}{\multicolumn{4}{|c|}{$\begin{array}{l}\text { 14. SUBJECT TERMS } \\
\text { Adjoint equations, Eigensolutions, Flow control, Flow stability, Vortex shedding }\end{array}$}} & $\begin{array}{l}\text { 15. NUMBEF OF PAGES } \\
10\end{array}$ \\
\hline & & & & $\begin{array}{r}\text { 16. PAICE CODE } \\
\text { A02 }\end{array}$ \\
\hline $\begin{array}{l}\text { 17. SECURITY CLASSIFICATION } \\
\text { OF REPORT } \\
\text { Unclassified }\end{array}$ & $\begin{array}{l}\text { 8. SECUUATY CLASSIFICATION } \\
\text { OF THIS PAGE } \\
\text { Unclassified }\end{array}$ & $\begin{array}{r}\text { 19. SEC } \\
\text { OF }\end{array}$ & ICATION & 20. LIMITATION OF ABSTRACT \\
\hline
\end{tabular}

